\title{
Coagulation of small grains in disks: the influence of residual infall and initial small-grain content
}

\author{
C. Dominik ${ }^{1,2}$ and C. P. Dullemond ${ }^{3}$ \\ 1 Sterrenkundig Instituut “Anton Pannekoek”, Kruislaan 403, 1098 SJ Amsterdam, The Netherlands \\ e-mail: dominik@science.uva.nl \\ 2 Afdeling Sterrenkunde, Radboud Universiteit Nijmegen, Postbus 9010, 6500 GL Nijmegen, The Netherlands \\ 3 Leader of Junior Research Group "Formation of planetary building blocks" at the Max-Planck-Institut für Astronomie, \\ Königstuhl 17, 69117 Heidelberg, Germany \\ e-mail: dullemon@mpia.de
}

Received 16 March 2008 / Accepted 4 August 2008

\begin{abstract}
Turbulent coagulation in protoplanetary disks is known to operate on a timescale far shorter than the lifetime of the disk. In the absence of mechanisms that replenish the small dust grain population, protoplanetary disks would rapidly lose their continuum opacitybearing dust. This is inconsistent with infrared observations of disks around T Tauri stars and Herbig Ae/Be stars, which are usually optically thick at visual wavelengths and show signatures of small $(a \lesssim 3 \mu \mathrm{m})$ grains. A plausible replenishing mechanism of small grains is collisional fragmentation or erosion of large dust aggregates, which model calculations predict to play an important role in protoplanetary disks. If optically thick disks are to be seen as proof for ongoing fragmentation or erosion, then alternative explanations for the existence of optically thick disks must be studied carefully. In this study we explore two scenarios. First, we study the effect of residual, low-level infall of matter onto the disk surface. We find that infall rates as low as $10^{-11} M_{\odot} / y r$ can, in principle, replenish the small grain population to a level that keeps the disk marginally optically thick. However, it remains to be seen if the assumption of such an inflow is realistic for star+disk systems at the age of several Myrs, at which winds and jets are expected to have removed any residual envelope. The effectiveness of even a low level infall can be understood by the strongly non-linear behavior of the coagulation equation: a high, fine-grain, dust density at any given time leads to very fast, effective removal of these small grains, while a low fine-grain density lasts for a far longer time. We then consider a second scenario in which, during the buildup phase of the disk, an intermediate fine-grain dust abundance is generated that is sufficiently low to ensure longevity of the state yet sufficiently high for the disk to remain optically thick. While our models confirm that such an "initial condition" can be constructed, we argue that these special initial conditions cannot be achieved during the disk build-up phase. In summary, fragmentation or erosion still appear to be the most promising processes to explain the abundant presence of small grains in old disks.
\end{abstract}

Key words. accretion, accretion disks - stars: circumstellar matter - stars: formation - stars: pre-main-sequence - infrared: stars

\section{Introduction}

The first stages of planet formation are now directly observable with infrared observations of protoplanetary disks. Most studies focus on the presence of slightly grown dust grains as evidence of the onset of this growth process (e.g. van Boekel et al. 2003; Przygodda et al. 2003; van Boekel et al. 2004; Kessler-Silacci et al. 2006). However, a much undervalued fact is that even old ( $\sim 3$ Myr or even older) disks still contain significant populations of small $(\lesssim 3 \mu \mathrm{m})$ grains as indicated by the presence of strong infrared solid state emission features (Malfait et al. 1998) and large optical depth (Bouwman et al. 2003). By optically thick, we refer to a significant optical depth as measured in the $V$ band, which causes the disk to absorb or scatter all incident stellar flux Models of dust coagulation without fragmentation predict consistently severe depletion in these small grains on timescale far smaller than the disk lifetime, as seen in calculations by Weidenschilling (1984), Dullemond \& Dominik (2005, hereafter DD05), and Tanaka et al. (2005, hereafter TIH05). While the assumption of a perfectly laminar disk may allow a small population of micron-size grains to survive (TIH05), even very weak turbulence (at a level of $\alpha=10^{-6}$ ) would enhance the coagulation process sufficiently for the disk to become optically thin.
In the above models, differential radial drift was not included: at low levels of turbulence, it is the dominant mechanism controlling the relative velocities of grains of various sizes (Whipple 1972; Weidenschilling 1977, 1997; Brauer et al. 2008), which implies that, without fragmentation, growth is enhanced by this mechanism.

Models of dust coagulation without a treatment of collisional fragmentation are of course unrealistic. Weidenschilling (1984, 1997) included fragmentation in his model. It turns out that fragmentation is a universal outcome. Models of particle motion in laminar and turbulent accretion disks predict collision velocities in excess of the critical value for break-up (Weidenschilling 1977; Völk et al. 1984; Blum \& Wurm 2000; for a review, see Dominik et al. 2007). Brauer et al. (2008) demonstrated that collisional fragmentation is a major barrier to the growth of dust aggregates beyond centimeter or decimeter sizes. However, it is still unknown if fragmentation can produce a sufficient amount of small grain dust to produce the disk opacity inferred from observations. This question is difficult to answer, because the amount of small grains produced in collisions is strongly dependent on collision parameters (Paszun \& Dominik, submitted to A\&A). It is therefore important to know if the opaqueness of protoplanetary disks is an indication of small grain production 
by fragmentation and erosion (as we argued in DD05), or if other mechanisms could retain the small grain population in disks, such as the thermal breakup of icy aggregates (Tanaka et al. 2005, poster at PPV), which can operate in a limited radial range.

In the present paper, we investigate if a continuous replenishment of small grains by residual infall of matter from the circumstellar envelope onto the disk's surface could sustain the small dust grain population. This infall might be residual infall from the parental cloud, or Bondi-Hoyle like accretion (Bondi \& Hoyle 1944) by a protoplanetary disk moving through a molecular cloud. We assume that the infall originates in the parental cloud, with zero velocity at infinity. As long as the motion of the star relative to the cloud is small compared with Keplerian velocities in the inner regions of the disk, these cases are similar. The idea of external replenishment was first studied by Mizuno et al. (1988), who were interested primarily in convection-driven turbulence (which requires optically-thick disks) during the early stages of the disk's lifetime. Using a simple one-zone model of a disk, they indeed showed that a mass infall rate of $10^{-6}$ and $10^{-7} M_{\odot} /$ yr could help to keep the optical depth of the disk high.

One can easily show that an envelope with an infall rate of $10^{-6} M_{\odot} /$ yr would be optically thick and completely obscure the star+disk system, while $10^{-7} M_{\odot} /$ yr would still be marginally optically thick. The typical star+disk systems studied in the context of grain growth and planet formation are "revealed sources" (class II in the Lada classification), meaning that they have envelopes that are optically thin. If this process is to work at all in these systems, it must work at relatively low infall rates, which we consider here.

In Sect. 3 we first study if the replenishment of the disk with small dust grains by an infalling tenuous envelope with low infall rate would be sufficient to ensure that the disk remained sufficiently optically thick to be consistent with observations. We employ the 1D vertical slice models for coagulation described by DD05, but with additional continuous infall of small grained dust at the top. We study the total vertical optical depth, the optical depth in grains smaller than $3 \mu \mathrm{m}$ (which are the silicatefeature-producing grains), and the optical depth in grains larger than $3 \mu \mathrm{m}$.

In Sect. 4 we consider the effects of a low initial abundance of small grains on the removal timescale of small grains. This involves models without external inflow, but with different dustto-gas ratios in the initial setup.

We discuss our results and present our conclusions in Sect. 5.

\section{The model}

\subsection{Disk and coagulation model}

We model the dust coagulation in a disk around a pre-mainsequence star. We begin the simulation at $t=0$, which represents the time immediately after the main build-up phase of the star+disk system. This is the moment when the main infall onto the system, which is of the order of $10^{-5} M_{\odot} / \mathrm{yr}$ during the buildup phase, either ceases entirely or reduces to far lower values. It is this much lower "residual infall" that we focus on in Sect. 3.

As our base star+disk model, we consider a $0.05 M_{\odot}$ disk around a $0.5 M_{\odot}$ star. The star is assumed to have an effective temperature of $4000 \mathrm{~K}$ and a radius of $2.5 R_{\odot}$. For the surface density of the disk, we assume a power law $\Sigma=\Sigma_{0}(r / \mathrm{AU})^{-1}$ (see e.g. Dullemond et al. 2007, for a review) where $r$ is the distance from the star. The disk radius is assumed to be $200 \mathrm{AU}$, and $M_{\text {disk }}=0.1 M_{*}=0.05 M_{\odot}$, such that the surface density at $1 \mathrm{AU}$ becomes $\Sigma_{0}=354 \mathrm{~g} / \mathrm{cm}^{2}$ (in gas). The vertical gas distribution is assumed to be in hydrostatic equilibrium, with a gas temperature given by a simple irradiation recipe $T_{\text {gas }}(r)=$ $(1 / \sqrt{2}) \phi^{1 / 4} T_{*} \sqrt{R_{\star} / r}$, where $\phi$ is the irradiation angle, which we assume to be 0.05 . The dust and gas are initially well-mixed, with a dust-to-gas ratio by mass of 0.01 . The dust grain size distribution in the initial disk model and in the infalling flow of matter is assumed to be an MRN distribution (Mathis et al. 1977) with particles distributed between 0.1 and $0.5 \mu \mathrm{m}$, following a power-law size distribution $f(a) \propto a^{-7 / 2}$. The dust grains are assumed to be astronomical silicate. The opacities were computed using Mie theory, with optical properties from Laor \& Draine (1993).

The coagulation model is that of DD05, which we used unmodified for these simulations. The disk's gas density and temperature were assumed to remain constant in spite of the vastly changing dust properties with time. We included coagulation by Brownian motion, vertical settling, and turbulent motions. The calculations do not include coagulation driven by radial drift in the disk. Radial drift is known to be important for the growth of large particles (Weidenschilling 1980, 1984). In the current paper, we are interested in the small grain component, for which the differences are expected to be minor. Furthermore, it allows us to compare directly with the results by DD05, who emphasized a need for some replenishment mechanism of small grains. In a model with strong turbulent mixing, the coagulation occurs particularly rapidly as material from the disk upper layers is mixed more deeply inside the disk, where the densities are higher and coagulation timescales are far shorter.

\subsection{Infalling envelope}

For the computations in this paper, we assume that the infalling matter is the residual influx from the collapse of a rotating cloud. The density and velocity distribution for this case were described with analytical models by Ulrich (1976), representing a simplified analysis of a the more general study by Terebey et al. (1984). The main parameters of this model are the infall rate $\dot{M}$ and the centrifugal radius $r_{\text {centr }}$, which we fixed to be $200 \mathrm{AU}$. The gas density at a distance $r$ and polar angle $\mu=\cos \theta$ is given by:

$\rho(r, \theta)=\frac{\dot{M}}{4 \pi \sqrt{G M r^{3}}}\left(1+\frac{\mu}{\mu_{0}}\right)^{-1 / 2}\left(\frac{\mu}{\mu_{0}}+\frac{2 \mu_{0}^{2} r_{\text {centr }}}{r}\right)^{-1}$

where $\mu_{0}$ is a solution of the equation:

$\frac{r}{r_{\text {centr }}}\left(1-\frac{\mu}{\mu_{0}}\right)=1-\mu_{0}^{2}$.

The $r$ - and $\theta$-velocity components are:

$$
\begin{aligned}
& v_{r}=-\left(\frac{G M}{r}\right)^{1 / 2}\left(1+\frac{\mu}{\mu_{0}}\right)^{1 / 2} \\
& v_{\theta}=\left(\frac{G M}{r}\right)^{1 / 2}\left(\frac{\mu_{0}-\mu}{\sqrt{1-\mu^{2}}}\right)\left(1+\frac{\mu}{\mu_{0}}\right)^{1 / 2} .
\end{aligned}
$$

Multiplying $v_{\theta}(\theta=\pi / 2)$ by the density $\rho(\theta=\theta / 2)$ provides the mass flux onto the disk surface:

$$
f_{\text {trickle }}=\frac{\dot{M}}{8 \pi r \mu_{0} r_{\text {centr }}}
$$

where $\mu=\sqrt{1-r / r_{\text {centr }}}$.

Some example values for the trickling inflow onto the disk surface are given in Table 1. 
Table 1. Values for various quantities at different distances $r$ from the star, for the model with trickling rate $10^{-9} M_{\odot} / \mathrm{yr}$.

\begin{tabular}{rrrrrrrrr}
\hline \hline $\begin{array}{c}r \\
\mathrm{AU}\end{array}$ & $\begin{array}{c}\Sigma \\
\mathrm{g} \mathrm{cm}^{-2}\end{array}$ & $\begin{array}{c}f_{\text {trickle }} \\
\mathrm{g} \mathrm{cm}^{-2} \mathrm{~s}^{-2}\end{array}$ & $\begin{array}{c}v_{\theta} \\
\mathrm{km} \mathrm{s}^{-1}\end{array}$ & $\begin{array}{c}T_{\text {mid }} \\
\mathrm{K}\end{array}$ & $H_{\mathrm{p}} / r$ & $z_{\text {sh }} / r$ & \multicolumn{1}{c}{$\begin{array}{c}t_{\text {stop }} \\
\text { days }\end{array}$} & $\Delta z / H_{\text {sh }}$ \\
\hline 1 & 354 & $5.6(-14)$ & 21 & 305 & 0.05 & 5.83 & 3.7 & 0.12 \\
10 & 35 & $5.8(-15)$ & 6.5 & 96 & 0.09 & 5.3 & 204 & 0.12 \\
100 & 3.5 & $8.0(-16)$ & 1.5 & 30 & 0.16 & 4.7 & 14600 & 0.12 \\
\hline
\end{tabular}

The quantities are: surface density $\Sigma$, trickling flux $f_{\text {trickle }}$, impact velocity of the infall $v_{\theta}$, midplane temperature $T_{\text {mid }}$, pressure scale height $H_{\mathrm{p}}$, accretion shock height $H_{\mathrm{sh}}$, stopping time for $0.1 \mu \mathrm{m}$ grains $t_{\text {stop }}$, deceleration length for $0.1 \mu \mathrm{m}$ grains in units of the shock height.

\subsection{Location of the accretion shock and dust injection}

As matter falls onto the disk, an accretion shock must form to decelerate the gas. The physics of the accretion shock is complex (e.g. Ruzmaikina \& Ip 1994), because the temperature behind the accretion shock has to be computed. In the current study we assume that the cooling behind the shock is fast, i.e. that the postshock gas quickly cools to the local temperature of the disk. We may then compute the location of the shock by equating the ram pressure in the infalling material to the local hydrostatic pressure in the disk and solve for the height above the disk. The ram pressure from the infalling material is given by

$p_{\text {ram }}=\rho(r, 0) v_{\theta}^{2}(r)$.

The hydrostatic pressure in the disk $p_{\text {disk }}$ is given as a function of height $z$ in the disk by

$p_{\text {disk }}=\gamma \rho_{\text {disk }} c_{\mathrm{s}}^{2}=\frac{\Sigma \gamma c_{\mathrm{s}}^{2}}{H_{\mathrm{p}} \sqrt{2 \pi}} \exp \left\{-\frac{z^{2}}{2 H_{\mathrm{p}}^{2}}\right\}$,

where $H_{\mathrm{p}}=c_{\mathrm{s}} / \Omega_{\mathrm{k}}$ is the pressure scale height in the disk, $c_{\mathrm{s}}=\sqrt{k T / \mu m_{\mathrm{p}}}$ is the sound velocity, $\mu$ is the mean molecular weight, $m_{\mathrm{p}}$ the proton mass, $\Omega_{\mathrm{k}}=\sqrt{G M_{\star} / r^{3}}$ is the local Kepler frequency, and $\gamma$ is the adiabatic index.

$z_{\mathrm{sh}}=\sqrt{-2 H_{\mathrm{p}} \ln \left[\frac{\sqrt{2 \pi} H_{\mathrm{p}} p_{\mathrm{ram}}}{\gamma c_{\mathrm{s}}^{2} \Sigma}\right]}$.

We find that this is typically 5 to 6 pressure scales above the disk (for detailed values, see Table 1).

When the infalling gas motion has stopped, the dust particles are injected into the disk with the free-fall speed at the considered location. Dust grains are decelerated over a certain distance, which we compute numerically. The results are reported in Table 1 and indicate that the stopping time is approximately a few hours, and the stopping length is far smaller than the disk scale height. Another issue is the temperature of the grains during deceleration. The grains heat up due to frictional coupling with the gas. We can estimate the heating with simple argument. The kinetic energy of the incoming dust grain is given by $0.5 m_{\mathrm{gr}} v_{\theta}^{2}$. Following the deceleration curve of the grain, we make the extreme assumption that the entire kinetic energy is transformed into heating grains (in reality, a large fraction will heat the surrounding gas). Equating this heating rate to a radiative cooling rate, we found that the grains were heated up by less than a few hundred degrees at $1 \mathrm{AU}$, and that the heating was negligible at 10 and $100 \mathrm{AU}$. The grain should therefore be able to reach the disk largely unchanged (e.g. Ruzmaikina \& Ip 1994).

We can therefore justify our simple treatment of dust injection. Dust particles are added to our uppermost grid cell in the local disk slice with an initial velocity of 0 . From that location, particles will settle down and become part of the coagulation process.

\section{Results I: effect of residual infall}

We completed the coagulation simulations at three different radii in the disk: $1 \mathrm{AU}, 10 \mathrm{AU}$ and $100 \mathrm{AU}$. We assumed three different infall rates $\left(0,10^{-11}, 10^{-7}\right) M_{\odot} / \mathrm{yr}$, which translate into local infall rates in $\mathrm{g} \mathrm{cm}^{-2} \mathrm{~s}^{-1}$ using Eq. (5). We also assumed two different strengths of turbulent mixing: a weakly turbulent disk ( $\alpha=10^{-6}$, as a nearly laminar case) and a strongly turbulent disk ( $\alpha=10^{-2}$, as typically found by MRI instability calculations, and also needed to explain the high accretion rates during the early stages of disk evolution). Here, $\alpha$ is the Shakura-Sunyaev parameter for viscosity, which can be used to parametrize the strength of the turbulence, assuming a Stokes number of unity. We do not include fully laminar conditions, since we believe that these are unrealistic. We allow each model to run for $1 \mathrm{Myr}$, in a few time-consuming exceptions we stopped earlier if a steady state had already been reached. This provides a $3 \times 4 \times 3$ matrix of models.

In Fig. 2, the resulting time-dependent optical vertical depths at $\lambda=0.55 \mu \mathrm{m}$ are shown for the case of $\alpha=10^{-6}$. The top row shows the results without residual infall, which can be most closely compared with the laminar models of DD05 and TIH05. It should be noted, however, that the small, but non-zero, turbulence in the present models has non-negligible effect on the results, and the comparison is therefore only partly valid. In these top three models, the coagulation is first driven by Brownian motion, which is responsible for the weak reduction in optical depth between $t=0$ and $t=300 \mathrm{yrs}$ for the $R=1$ AU case. The optical depth then suddenly begins to drop, as sedimentationdriven coagulation reaches its peak intensity and efficiently removes small grains from the disk. The slow further decline in optical depth is then caused by turbulence-driven coagulation, which slowly removes approximately 0.1 percent fine dust that escaped the sweep-up by settling grains. The final optical depth at 1 Myr turns is 0.2 at $1 \mathrm{AU}, 2$ at $10 \mathrm{AU}$, and 12 at $100 \mathrm{AU}$. While the outer regions of the disk are still optically thick, the inner regions have become optically thin. This was found by TIH05 to occur in their laminar models and they proposed that this might be a possible mechanism for explaining "inner holes" in certain disks (e.g. Forrest et al. 2004; Bouwman et al. 2003; Calvet et al. 2002). The second row of Fig. 1 shows the same models, but with a continuous trickling infall at a (global) rate of $\dot{M}=10^{-11} M_{\odot} / \mathrm{yr}$. We see that at $1 \mathrm{AU}$ this causes the optical depth to become constant at a value of 2 . The outer disk is hardly affected. At an infall rate of $\dot{M}=10^{-7} M_{\odot} / y r$, lower row, the optical depth at $1 \mathrm{AU}$ becomes constant at 80 and at $10 \mathrm{AU}$ and $100 \mathrm{AU}$ at 70. These values are sufficiently high for the disk to be considered optically thick disk.

Figure 2 shows the same models as Fig. 1 but now with $\alpha=$ $10^{-2}$. Again, the top row shows the models without infall. As for the low- $\alpha$ case, Brownian motion produces an initially slow decline in the optical depth up to the onset of sedimentationdriven coagulation. However, in contrast to the low- $\alpha$ case, the 


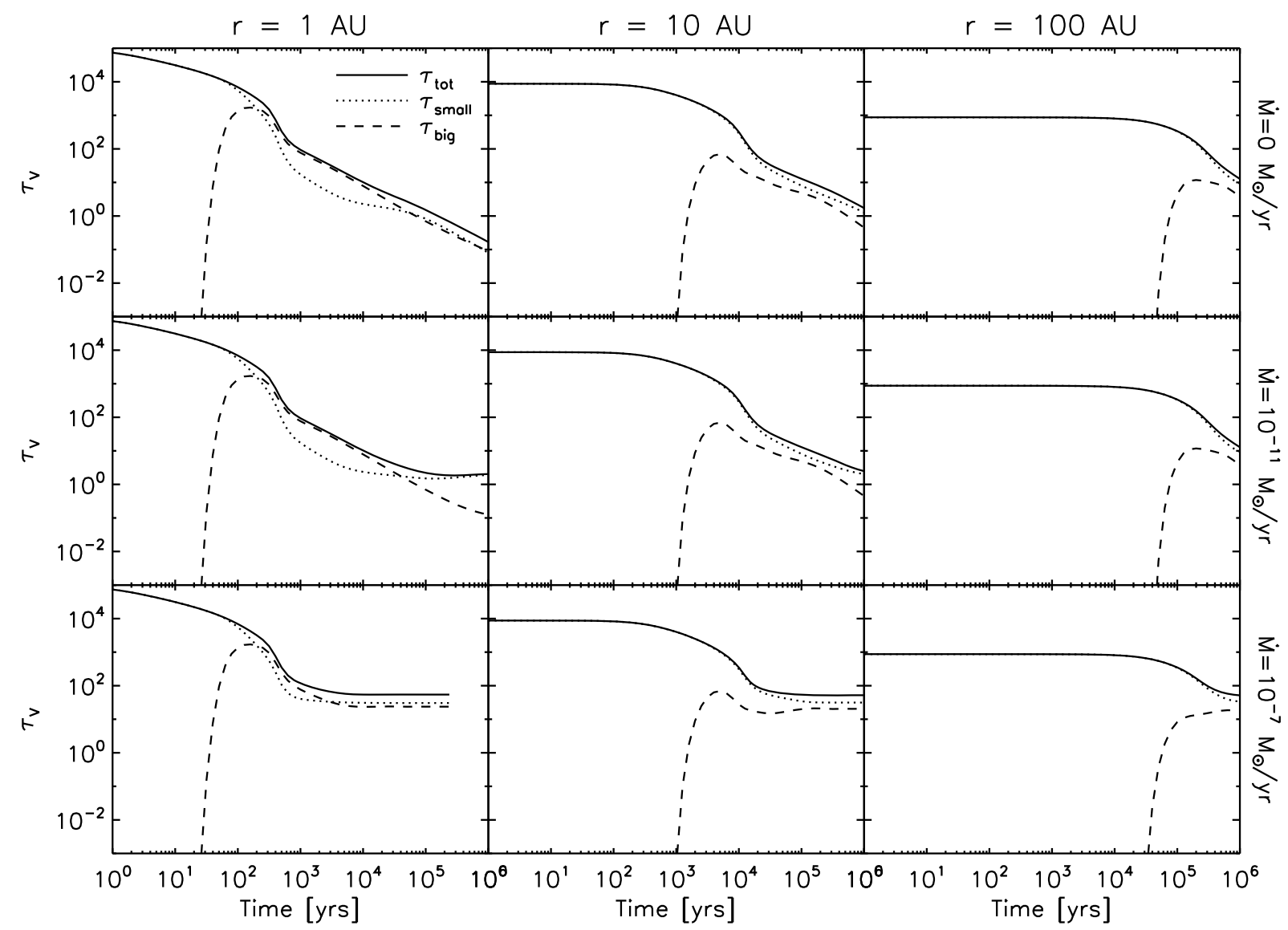

Fig. 1. The vertical $V$-band optical depth of the disk with turbulent parameter $\alpha=10^{-2}$ at $1 \mathrm{AU}, 10 \mathrm{AU}$ and $100 \mathrm{AU}$ (from left to right) and infall rates $0,10^{-11} M_{\odot} / \mathrm{yr}, 10^{-7} M_{\odot} / \mathrm{yr}$ (from top to bottom). The dotted line is the contribution to the optical depth by grains with radius $\leq 3 \mu \mathrm{m}$, the dashed line is the contribution by grains $>3 \mu \mathrm{m}$.

subsequent decline in optical depth is extremely fast, which differs from our results in Fig. 1. Turbulence-driven coagulation is far stronger than in the $\alpha=10^{-6}$ case, and becomes highly effective in removing small grains. In these simulations we emphasize that the optical depth at late times is due primarily to particles larger than $3 \mu \mathrm{m}$, an effect that might be artificial and in part caused by the neglect of grain drift, unlike the low- $\alpha$ models. In the second row, we see that at $1 \mathrm{AU}$ and $10 \mathrm{AU}$ the trickling infall of fresh matter leads to a slow recovery in the optical depth, after its strong previous decline. The optical depth is now primarily controlled by small particles. At $1 \mathrm{AU}$, the optical depth levels off at around 1, and at $10 \mathrm{AU}$ becomes 0.1 , which is still optically thin, and lower than at $1 \mathrm{AU}$. At this radius, the optical depth is still increasing after 1 Myr. At 100 AU the infall has not produced any noticeable effect. In the last row, we can see that following the recuperation of the optical depth after its reduction it appears to saturate at a value of 150 at $1 \mathrm{AU}$ as well as at $10 \mathrm{AU}$. The system reaches an equilibrium state, in which the rate of fine dust removal appears to equal the rate of replenishment by fresh fine dust. The equilibrium state is still imperfect, since the large grain population continues to oscillate, as can be seen by the dashed lines. However, since these large grains ( $>3 \mu \mathrm{m}$ ) represent only a small contribution to the optical depth, this is of no relevance to the optical depth issue. Even at $100 \mathrm{AU}$, we now see that the replenishment by new grains is effective and increases the optical depth, but an equilibrium state is not yet reached.

Judging from these results, we see that, in principle, even a small residual infall rate onto the disk can explain the observed optical thickness of $\mathrm{T}$ Tauri star disks, even if fragmentation is not active and therefore cannot replenish the population of small grains. Whether infall scenarios with these rates in the late evolutionary stages are realistic is, however, still a major issue of concern, which we discuss in Sect. 5.

\section{Results II: effect of reduced initial fine-dust abundance}

In the models of coagulation described here, it is seen that the dust in the disk coagulates initially extremely rapidly, reducing the optical depth to almost zero apart from that due to a trickling accretion of new material. It is striking, however, that even a very low residual infall rate of, say, $10^{-11} M_{\odot} / \mathrm{yr}$ can have this effect. At first sight this appears difficult to understand, because at this trickling rate it takes 5 Gyr to replenish fully a disk of $0.05 M_{\odot}$, whereas the removal of small grains occurs within $10^{4}$ years. One would expect the replenishment to be unable to compete with the coagulation. The speed of coagulation is however inversely proportional to the density of dust. Therefore, the time to grow to sizes that no longer contribute to the optical depth will be inversely proportional to the density of fine-grain material. If we begin with more fine-grained dust, we therefore end with less.

This suggests another way to retain the value the optical depth in a disk for a long time. Our simulations have consistently started from an already constructed disk, i.e. after the early build-up phase of the disk. Without justification, we have assumed that at this point in time the dust population is interstellar 


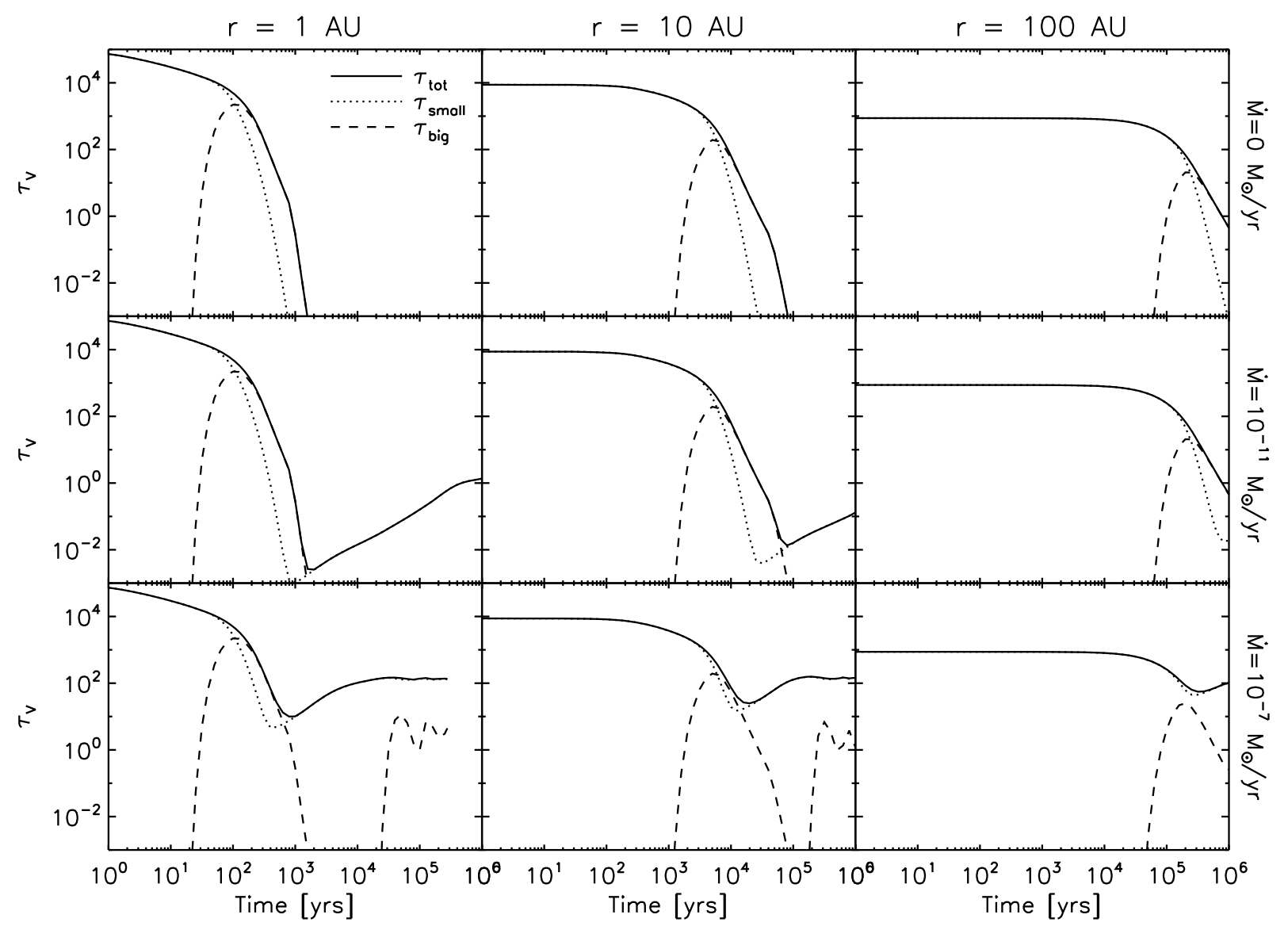

Fig. 2. Like Fig. 2, but with with turbulent parameter $\alpha=10^{-6}$.

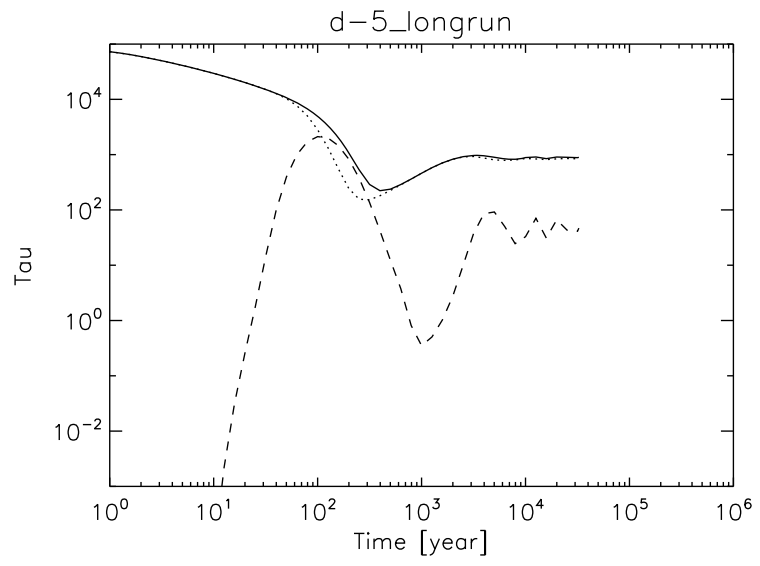

Fig. 3. The time-dependent optical depth for the case when we apply our model to the build-up phase of the disk, with an infall rate of $10^{-5} M_{\odot} /$ yr. Since this simulation is merely meant as an "initial condition" for the Myr-long coagulation process studied in this paper, we run it only until an equilibrium state has set in. As can be seen in this figure, this happens after about $10^{4}$ years.

in both size distribution and abundance. However, coagulation is also active during the build-up phase of the disk (Suttner et al. 1999; Suttner \& Yorke 2001), and it is therefore to be expected that by the time the main infall phase is over (and our simulation begins) the abundance of fine-grain dust has already decreased.

To investigate the significance of this reduction, we could apply the models of Sect. 3 to the build-up phase, and observe the equilibrium state that is reached. We simply need to adopt a far higher infall rate, i.e. a rate at which a star can be built in about $0.1 \mathrm{Myr}$. In our case this corresponds to an infall rate of about $10^{-5} M_{\odot} / y$. In Fig. 3, we see that, at these high infall rates, the equilibrium is reached at a very early stage, justifying the use of this state as an initial condition. In this particular case the figure shows that the optical depth is reduced by about 2 orders of magnitude compared to the "interstellar" dust abundance case. As an initial condition of our Myr-long simulation, it would therefore be justifiable to begin with a fine-dust abundance that is a factor of 100 below the interstellar medium value. However, we have not modeled this buildup phase consistently, and more sophisticated physical ingredients, such as pre-collapse coagulation, disk accretion, and radial drift of larger grains, would need to be included, which is well beyond the scope of this paper. We therefore study the effect of a reduced initial fine-dust abundance on the Myr-long simulations, by using the initial abundance as a free parameter. This is implemented by completing models without any residual infall, but with decreased initial dust-to-gas ratio. We ignore any population of very large grains that may have been produced during the build-up phase.

The results displayed in Fig. 4 indicate that the initial optical depth is proportional to the initial dust-to-gas ratio (which is obvious) and that this optical depth can be sustained for a time that is inversely proportional to the initial dust-to-gas ratio. For example, with an interstellar dust-to-gas ratio (i.e. $10^{-2}$ ) the optical depth is almost $10^{5}$ at first, but a steep decrease occurs after 100 years. If we begin the simulation, however, with a dust-togas ratio of $10^{-7}$ (i.e. a factor of $10^{5}$ lower than interstellar), then the initial optical depth is close to 1 and can be sustained 


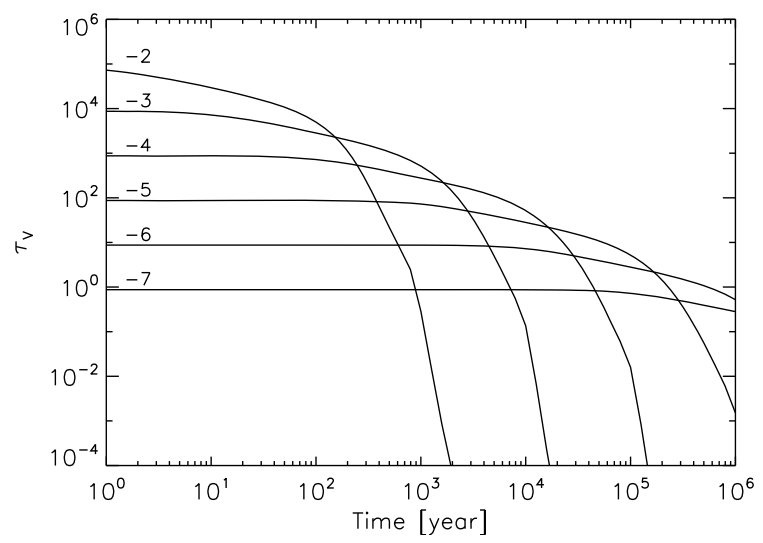

Fig. 4. The time-dependent optical depth for the for disks with varying initial dust-to-gas ratio. The curves are labeled with $\log f_{\mathrm{dg}}$. This figure clearly demonstrates the "The higher you start, the lower you end" effect caused by the non-linear behavior of the coagulation equation.

for about 1 Myr. It therefore appears that the strong reduction of optical depth may occur as late as $1 \mathrm{Myr}$, but only if start with an optical depth that is already close to 1 .

This implies that there exists a small region of parameter space in which the disk can remain marginally optically thick $\left(\tau_{V} \simeq 1\right)$ for $1 \mathrm{Myr}$, without fragmentation or any other means of replenishment of small grains. However, many disks live even longer, and there is a large volume of observational evidence suggesting that these disks have higher optical depths (e.g. Forrest et al. 2004, and Dullemond \& Dominik 2004; for a review of fitting disk models to observations, see Watson et al. 2007).

It will also be difficult to produce this specific initial state, for the following reason: low optical depth equilibrium states require a long time to be established. The equilibrium state reached during the disk build-up phase is defined to be the state in which the timescale of replenishment of fine dust equals the timescale of removal of fine dust by coagulation. Therefore, the build-up time of the state with replenishment equals the lifetime of the state without replenishment. For example, during the disk buildup phase (clearly at a low infall rate) we would require $1 \mathrm{Myr}$ to achieve an equilibrium state that could, after the end of infall, be sustained for another $1 \mathrm{Myr}$ as an optically thick disk. It is believed, however, that the build-up phase of a star+disk system (i.e. the class $\mathrm{O}$ and class I phases in the Lada/Andre scheme) has a duration of less than 1 Myr (Andre \& Montmerle 1994; Barsony 1994), implying that this phase is significantly shorter than the typical disk lifetime. The conclusion is that the dust cannot reach a steady state that lasts for a few Myrs. Any steady state reached during this time is certain to decay shortly after the main infall phase. It is also possible that no steady state of the dust population is reached at all during this disk build-up phase. Both situations are not promising for keeping the optical depth high over a few Myrs time.

\section{Discussion}

\subsection{Consequences for dust fragmentation}

In Sect. 4, we have shown that a low value of fine-grained dust density in the disk can in principle persist for a long time, but that there is only a narrow range in parameter space for which the disk optical depth remains above unity for a few million years. It also takes a long time to reach this long-lasting state. This result is fully independent of the mechanism that produces the small grains in the disk. We can also apply it to small grains produced by aggregate fragmentation and erosion (instead of trickling infall). If, after studying possible alternatives in this paper, we return to fragmentation and erosion as the most likely sources of small grain replenishment in disks, then we must conclude that these must be ongoing processes lasting throughout the fewmillion-year lifetime of the disk. If this replenishment process were to switch off for some reason, the disk would quickly become optically thin.

\subsection{Limits on infall onto T Tauri star disks}

How realistic is the assumption of low-level infall onto the disk? There are several ways to place constraints using both observational and theoretical arguments. In the following, we show that acceptable infall rates are constrained to be in the narrow range between $10^{-7}$ and $10^{-8} M_{\odot} / \mathrm{yr}$.

\subsubsection{Optical depth of the envelope}

We emphasize that we consider sources with only limited extinction toward the star. Many of the sources with optically thick disks have circumstellar reddening $A_{V}$ lower than (sometimes far lower than) two (e.g. van den Ancker et al. 1997). The largest contribution to the optical depth of the infalling envelope originates in the region around $r \simeq r_{\text {centr }}$. Using the density distribution from the Ulrich infall model, we arrive at an optical depth in the visual of 1.88 for an infall rate of $4 \times 10^{-7} M_{\odot} / y$. Therefore, the maximum infall rate that is consistent with the class II status of these sources is about this value. The optical depth at different infall rates obviously scales linearly with the infall rate.

\subsubsection{Stellar winds}

Young stars are also known to possess stellar winds, which can remove residual circumstellar envelopes. Using infrared and UV lines, Dupree et al. (2005) argued that TW Hya accelerates a hot wind with a velocity of $400 \mathrm{~km} \mathrm{~s}^{-1}$ and a mass loss rate of about $10^{-11} M_{\odot} / y r$, which should be able to quench any infall with rates below $10^{-9} M_{\odot} / y r$. The evidence for this wind was however questioned by Johns-Krull \& Herczeg (2007). If these wind properties were typical for T Tauri stars, $10^{-9} M_{\odot} /$ yr would be a lower limit for residual infall to reach the disk.

\subsubsection{Radiation pressure and dust/gas separation}

Another obstacle to any residual infall is radiation pressure. At low infall rates, the envelope is optically thin and the radiation reaches all the dust grains in the infalling gas. The problem of radiative blowout of grains from main-sequence stars was studied extensively in the context of the Vega phenomenon and for $\beta$ meteorites in the Solar System (Burns et al. 1979; Gruen \& Zook 1980; Artymowicz 1988; Krivova et al. 2000). For the luminosity of the Sun, a small range of dust particle sizes around $0.1 \mu \mathrm{m}$ have the correct properties for radiation pressure to exceed gravitational forces (Burns et al. 1979). For more luminous sources such as A stars or even their still more luminous Herbig Ae precursors, the blowout limit is pushed to $10 \mu \mathrm{m}$ and beyond, such that grains in a size range from 0.01 to $10 \mu \mathrm{m}$ should be blown away from the star (Artymowicz 1988). The one difference in the scenario considered here is of course that the dust grains are embedded in an infalling gas envelope, which is not affected by any 
significant radiation pressure and could possibly drag the dust particles along.

The drift velocity caused by the radiation pressure on dust grains must then exceed the infall velocity of the material. We can place a limit on the mass accretion rates necessary for dragging the grains along in the following way. In a spherical free-fall accretion scenario with a constant infall rate $\dot{M}$, the local velocity is given by $v(r)=-\sqrt{2 G M_{\star} / r}$ and the gas density is given by $\rho=\dot{M} /\left(4 \pi r^{2}|v|\right)$. We assume that a dust grain is embedded in that infall. The stellar radiation in incident on the grain with a flux $L_{\star} /\left(4 \pi r^{2}\right)$. The radiation force on the grain is then

$F_{\text {rad }}=\frac{L_{\star}}{4 \pi r^{2} c} Q_{\mathrm{pr}} \pi a^{2}$

where $a$ is the grain radius and $c$ is the speed of light. The efficiency for pressure transmission to the grain is $Q_{\mathrm{pr}}=Q_{\mathrm{abs}}+$ $Q_{\text {scat }}\langle\cos \alpha\rangle$ is, with $Q_{\text {abs }}$ being the absorption efficiency, $Q_{\text {scat }}$ the scattering efficiency, and $\langle\cos \alpha\rangle$ the angular average of the scattering phase function (Bohren \& Huffman 1983). $Q_{\mathrm{pr}}$ is dependent on the grain size. In Herbig AeBe stars with a radiation temperature of $10000 \mathrm{~K}, Q_{\mathrm{pr}}$ will be of order 1 for all grain sizes considered here, whereas in the cooler radiation field of $\mathrm{T}$ Tauri stars, it will be of order 1 for $a \geq 1 \mu \mathrm{m}$, and somewhat smaller for $0.1 \mu \mathrm{m}$ grains. The grains will not move toward the star if the radiation force can at least balance the drag force of the gas streaming toward the star. The limiting case is reached if the grain is at rest with respect to the star. Then the gas streams by at a velocity $v=v_{\mathrm{ff}}$. As the free-fall flow is supersonic, we must apply the friction law for the supersonic case in which we have

$F_{\text {fric }}=-\pi a^{2} \rho v^{2}$.

To keep the grain fixed in space, the friction force must be balanced by the radiation force,

$F_{\text {fric }}+F_{\text {rad }}=0$

where we are neglecting the gravitational force on the dust grains, restricting the discussion to grains that are clearly inside the blow-out size regime. This equality is satisfied for a critical mass accretion rate

$$
\begin{aligned}
\dot{M}_{\mathrm{cr}} & =\frac{3 L_{\star} Q_{\mathrm{pr}} \sqrt{r}}{\sqrt{2 G M_{\star}} c} \\
& =4.8 \times 10^{-8} \frac{M_{\odot}}{\mathrm{yr}} \frac{L_{\star}}{L_{\odot}} \frac{Q_{\mathrm{pr}}}{1} \sqrt{\frac{M_{\odot}}{M_{\star}}} \sqrt{\frac{r}{10000 \mathrm{AU}}} .
\end{aligned}
$$

As we can see, the mass loss rate required to drag dust grains along is a function of distance from the star. Far away from the star, a relatively high mass infall rate will be required for the dust grains to be dragged along. As the infall moves closer to the star, it becomes easier to drag the dust grains along. Therefore, the true limiting infall rate is determined at large distances, where the free fall toward the star begins. If the infall starts at $10000 \mathrm{AU}$ from the star, we find a minimum infall rate of $4.8 \times 10^{-8} M_{\odot} / \mathrm{yr}$ for solar parameters.

It is therefore clear that gas drag may help to overcome the outward pushing radiation force only for the highest infall rates. For Herbig AeBe stars, the infall rates resulting from our estimate contradict directly the observations of low $A_{V}$, and imply that low-level infall cannot play a role. For T Tauri stars, there is a window of infall rates between approximately $4 \times 10^{-7}$ and $5 \times 10^{-8} M_{\odot} / \mathrm{yr}$, which in principle would allow accretion of dust grains onto the disk.

\subsection{General remarks}

In this paper we have studied two scenarios to keep the disk optically thick in spite of dust coagulation.

The first scenario is the low rate of matter infall onto the disk. This could help to retain a sufficiently high optical depth. However, it is unclear if these infall rates are in fact present. We can place severe limits on the allowed rates by considering the optical depth of the infalling envelope, and the effects of stellar winds and radiation pressure on dust (see Sect. 5.2).

The second scenario involved beginning the coagulation simulation with a reduced abundance of fine dust. We have justified these special initial conditions with ongoing coagulation during disk build-up. However, we have concluded that the special initial state needed to sustain $\tau_{V} \gtrsim 1$ without dust replenishment cannot be achieved in the time available. Even if this were achievable, the optical depth that one can sustain for 1 Myr does not exceed $\tau_{V} \simeq 1$, in contradiction with observational evidence.

Therefore, fragmentation and erosion of aggregates appear still to be the most promising explanation for the observed small dust grain populations in disks of $1 \mathrm{Myr}$ age or older, as proposed by DD05.

It should be noted that the models we have presented in this paper are based on considerable simplifications. The most important of these is that we do not include the accretion of gas in the disk, toward the star. Any material that is located at $1 \mathrm{AU}$ will be advected toward the star on a viscous timescale, which for a turbulence of $\alpha=10^{-2}$ (assuming that the Schmidt number is 1) equals $10^{4}$ years, and the structure of the disk may evolve significantly over a period of $1 \mathrm{Myr}$. As stated before, we have not included radial drift of grains. It is clear that the next step will have to be the full time-dependent modeling of the coupled equations of disk formation and evolution, and dust coagulation. With such models one could repeat the study completed here in a fully consistent way.

\section{Conclusion}

With this study, we arrive at the following conclusions:

1. Low-level infall onto a disk can have a surprisingly strong influence on the steady-state abundance of small dust grains in a protoplanetary disk, in particular if the infall reaches a disk that is initially cleared of small grains. This is due to a strong non-linear effect: the high initial dust content of the disk leads to very fast coagulation and a complete loss of small grains. The low dust densities produced by constant trickling are balanced by a far slower coagulation.

2. To be significant, the trickling rate must exceed $10^{-11} M_{\odot} / \mathrm{yr}$. Over a lifetime of $1 \mathrm{Myr}$, this corresponds to a total trickling mass in gas of $10^{-5} M_{\odot}$.

3. In our calculations, the optical depth of the disk is produced by grains smaller than $3 \mu \mathrm{m}$. In this case, we expect that the $10 \mu \mathrm{m}$ feature, an important indicator for small grains, will be present. Radiative transfer calculations will be required to determine whether this is the case.

4. Almost perfectly laminar disks can remain marginally optically thick even without trickling, but for these disks the optical depth is produced by large grains $(>3 \mu \mathrm{m})$.

5. Radiation pressure in Herbig stars excludes trickling as a viable mechanism for keeping the disk optically thick. Even for T Tauri stars, mass infall rates higher than $10^{-8} M_{\odot} / \mathrm{yr}$ are needed to overcome the effects of radiation pressure on small grains. Large grains (above the blow-out limit) can be 
accreted more easily. For Herbig stars, the size limit of the order of $a \gtrsim 10 \mu \mathrm{m}$, rendering it useless. In T Tauri stars, we find $a \gtrsim 1 \mu \mathrm{m}$, which is dependent on luminosity.

6. Low initial dust-to-gas ratios could lead to disks with longlasting optical depth of order unity. However, it appears unlikely that these states correspond to initial conditions because the time required to achieve these states is comparable to their duration.

The above conclusions can be summarized in the following way: While a low-level trickling infall of residual envelope matter onto the disk might conceivably sustain the disks's optical depth above unity even without grain fragmentation, the conditions for this residual infall are very stringent. Ongoing and perpetual aggregate fragmentation and erosion in the disk as an explanation for the disk's optical depth remains a more effective explanation.

Acknowledgements. C.P.D. acknowledges financial support from the Max Planck Gesellschaft under the SNWG program. C.D. would like to thank the Dutch Top Research School NOVA (network 2) for financial support and the Leids Kerkhoven-Bosscha Fonds for travel support. We thank G. Wurm for a discussion about the effect of initial dust densities which has in part triggered this work.

\section{References}

Andre, P., \& Montmerle, T. 1994, ApJ, 420, 837

Artymowicz, P. 1988, ApJ, 335, L79

Barsony, M. 1994, in Clouds, Cores, and Low Mass Stars, ed. D. P. Clemens, \& R. Barvainis, ASP Conf. Ser., 65, 197

Blum, J., \& Wurm, G. 2000, Icarus, 143, 138

Bohren, C. F., \& Huffman, D. R. 1983, Absorption and Scattering of Light by Small Particles (New York: John Wiley \& Sons)

Bondi, H., \& Hoyle, F. 1944, MNRAS, 104, 273

Bouwman, J., de Koter, A., Dominik, C., \& Waters, L. B. F. M. 2003, A\&A, 401, 577
Brauer, F., Dullemond, C. P., \& Henning, T. 2008, A\&A, 480, 859

Burns, J. A., Lamy, P. L., \& Soter, S. 1979, Icarus, 40, 1

Calvet, N., D’Alessio, P., Hartmann, L., Wilner, D., Walsh, A., \& Sitko, M. 2002, ApJ, 568, 1008

Dominik, C., Blum, J., Cuzzi, J. N., \& Wurm, G. 2007, in Protostars and Planets V, ed. B. Reipurth, D. Jewitt, \& K. Keil, 783

Dullemond, C. P., \& Dominik, C. 2004, A\&A, 417, 159

Dullemond, C. P., \& Dominik, C. 2005, A\&A, 434, 971

Dullemond, C. P., Hollenbach, D., Kamp, I., \& D'Alessio, P. 2007, in Protostars and Planets V, ed. B. Reipurth, D. Jewitt, \& K. Keil, 555

Dupree, A. K., Brickhouse, N. S., Smith, G. H., \& Strader, J. 2005, ApJ, 625, L131

Forrest, W. J., Sargent, B., Furlan, E., et al. 2004, ApJS, 154, 443

Gruen, G., \& Zook, H. A. 1980, in Solid Particles in the Solar System, ed. I. Halliday, \& B. A. McIntosh, IAU Symp., 90, 293

Johns-Krull, C. M., \& Herczeg, G. J. 2007, ApJ, 655, 345

Kessler-Silacci, J., Augereau, J.-C., Dullemond, C. P., et al. 2006, ApJ, 639, 275

Krivova, N. A., Krivov, A. V., \& Mann, I. 2000, ApJ, 539, 424

Laor, A., \& Draine, B. T. 1993, ApJ, 402, 441

Malfait, K., Waelkens, C., Waters, L. B. F. M., et al. 1998, A\&A, 332, L25

Mathis, J. S., Rumpl, W., \& Nordsieck, K. H. 1977, ApJ, 217, 425

Mizuno, H., Markiewicz, W. J., \& Voelk, H. J. 1988, A\&A, 195, 183

Przygodda, F., van Boekel, R., Àbrahàm, P., et al. 2003, A\&A, 412, L43

Ruzmaikina, T. V., \& Ip, W. H. 1994, Icarus, 112, 430

Suttner, G., \& Yorke, H. W. 2001, ApJ, 551, 461

Suttner, G., Yorke, H. W., \& Lin, D. N. C. 1999, ApJ, 524, 857

Tanaka, H., Himeno, Y., \& Ida, S. 2005, ApJ, 625, 414

Terebey, S., Shu, F. H., \& Cassen, P. 1984, ApJ, 286, 529

Ulrich, R. K. 1976, ApJ, 210, 377

van Boekel, R., Waters, L. B. F. M., Dominik, C., et al. 2003, A\&A, 400, L21

van Boekel, R., Min, M., Leinert, C., et al. 2004, Nature, 432, 479

van den Ancker, M. E., The, P. S., Tjin A Djie, H. R. E., et al. 1997, A\&A, 324, L33

Watson, A. M., Stapelfeldt, K. R., Wood, K., \& Ménard, F. 2007, in Protostars and Planets V, ed. B. Reipurth, D. Jewitt, \& K. Keil, 523

Weidenschilling, S. J. 1977, MNRAS, 180, 57

Weidenschilling, S. J. 1980, Icarus, 44, 172

Weidenschilling, S. J. 1984, Icarus, 60, 553

Weidenschilling, S. J. 1997, Icarus, 127, 290

Whipple, F. L. 1972, in From Plasma to Planet, ed. A. Elvius, 211 